\title{
miR-98 inhibits cell proliferation and induces cell apoptosis by targeting MAPK6 in HUVECs
}

\author{
CHUANXIAN HU, SU HUANG, FAFU WU and HUI DING \\ Cardiopulmonary Surgery, Huai'an First People's Hospital, Jiangsu, Huai'an 223300, P.R. China
}

Received May 15, 2017; Accepted November 28, 2017

DOI: $10.3892 /$ etm.2018.5735

\begin{abstract}
The aim of current study was to explore the role of microRNA (miR)-98 in atherosclerosis. Human vascular endothelial cells (HVECs) were isolated from the peripheral blood of healthy volunteers and patients with atherosclerosis. Compared with endothelial cells from the healthy control group, the expression level of mitogen activated protein kinase (MAPK)6 was significantly upregulated and miR-98 was downregulated in the endothelial cells of patients with atherosclerosis. The human umbilical vein endothelial cell line (HUVEC) was adopted to perform in vitro studies. Overexpression of miR-98 reduced the proliferation and induced the apoptosis of HUVECs, which were revealed using an MTT assay, and flow cytometry assay, respectively. The aforementioned influences of miR-98 on HUVECs were mediated by targeting MAPK6, which was verified using luciferase assays. Additionally, the overexpression of miR-98 reduced the protein level of apoptosis regulator Bcl-2 and MAPK6; however, it induced the protein expression of caspase- 3 and apoptosis regulator Bax. In conclusion, these findings demonstrate that miR-98 is an important regulator of atherosclerosis, suggesting that miR-98 may be a potential therapeutic target for the treatment of atherosclerosis.
\end{abstract}

\section{Introduction}

Atherosclerosis, a unique form of arteriosclerosis, presents as arterial wall thickening due to the invasion/accumulation of white blood cells and proliferation of intimal smooth muscle cell $(1,2)$. Vascular endothelium contributes to the development of atherosclerosis via abnormal cell proliferation and apoptosis (3). Endothelial cells maintain the homeostasis of the vascular system (4). However, the precise molecular mechanisms which underlie the contributions of the vascular endothelium to atherosclerosis remain unclear and complex.

Correspondence to: Dr Hui Ding, Cardiopulmonary Surgery, Huai'an First People's Hospital, 6 West Beijing Road, Jiangsu, Huai'an 223300, P.R. China

E-mail: dinghui1152@sina.com

Key words: atherosclerosis, miR-98, MAPK6
After binding to the 3' untranslated region (UTR) of target genes, microRNAs (miRNAs, miR) repress protein expression levels by mRNA destabilization or/and translation inhibition (5). Recent studies also found that miRNAs had the ability to reduce mRNA targets by binding to 5'UTR or protein-coding exons $(6,7)$. Circulating miRNAs serve critical roles in multiple diseases, including atherosclerosis (8) and cancer (9), thus showing enormous potential as novel biomarkers for various diseases. Consequently, it has emerged that miRNAs function as epigenetic regulators in diverse biological processes, including cell proliferation (10) and tumorigenesis (11). In diseased vascular vessels, dysregulation of miRNAs, for instance, miR-33 (12) and miR-133 (13), has been detected.

Mitogen activated protein kinase 6 (MAPK6) is an enzyme which is encoded by the MAPK6 gene in humans (14). MAPK6 has been shown to modulate cell migration, proliferation and angiogenesis in primary human umbilical vein endothelial cells (HUVECs), and has been reported to play a key role in maintaining normal physiological conditions of vascular smooth muscle cells (VSMCs) (15). In addition, in a previous study, the knockdown of MAPK6 damaged tube formation by VSMCs (16).

However, it remains unclear whether MAPK6 could be regulated by miRNA in the functions of the vascular endothelium. The current study aimed to clarify this.

\section{Patients and methods}

Participants. All study participants provided written informed consent. The current study was approved by the Ethics Committee of the Huai'an First People's Hospital (Jiangsu, China). Sixty atherosclerotic patients (mean age, 60.6 years; 36 male and 24 female) and 51 healthy volunteers (mean age, 56.5 years; 31 male and 20 female) were enrolled in the Huai'an First People's Hospital. Human vascular endothelial cells (HVECs) were isolated from the peripheral blood of healthy volunteers and patients with atherosclerosis using a human vascular endothelial cell separation medium kit (Sangon Biotech, Shanghai, China). HVECs were used for detection of the differences of MAPK6/miRNAs between healthy volunteers and patients with atherosclerosis.

Cell culture and transfection. HUVECs were used for the conducting of the following in vitro experiments. Briefly, HUVEC cell dissolution was performed by keeping in $37^{\circ} \mathrm{C}$ 
for $5 \mathrm{~min}$, transferred into a centrifuge tube and centrifuged (125 g, 5-7 min). HUVEC cell retrieved was balanced by RPMI (Lonza, Allendale, NJ, USA), 10\% fetal bovine serum (Sigma, St. Louis, MO, USA) and inoculated on $60 \times 20 \mathrm{~mm}$ Petri dishes $\left(1.3 \times 10^{5}\right.$ cells $\left./ \mathrm{ml}\right)$. Endothelial cells were cultivated in $5 \mathrm{ml}$ culture medium, inoculated in flasks overnight at $37^{\circ} \mathrm{C}<5 \% \mathrm{CO}_{2}$ with culture media changing every other day. When cellular density reached $80 \%$, passaging was performed at passaging rates of 1:2 or 1:3. After treatment of trypsin, endothelial cells in suspension were centrifuged, the obtained pellets were re-suspended in culture medium and inoculated on new Petri dishes.

HUVECs were seeded in Dulbecco's modified Eagle's medium containing $10 \%$ fetal bovine serum, $1 \% 100 \mathrm{U} / \mathrm{ml}$ penicillin and $1 \% 100 \mathrm{mg} / \mathrm{ml}$ streptomycin, thereafter, cultured in an incubator with $5 \% \mathrm{CO}_{2}$ at $37^{\circ} \mathrm{C}$.

As for cell transfection, hsa-miR-98 mimic or hsa-miR-negative control (NC) mimic was transfected into HUVECs by opti-MEM and Lipofectamine 2000 (Invitrogen, Carlsbad, CA, USA) in accordance with manufacturer's instructions.

Luciferase reporter assays. The binding site between hsa-miR-98 and MAPK6 was predicted by miRanda. For luciferase reporter assay, HUVECs were seeded in 24-well plates, afterwards, HUVECs were transfected with WT or mutant reporter plasmid and hsa-miR-98 mimic or hsa-miR-NC mimic by Lipofectamine 2000. After $24 \mathrm{~h}$, dual luciferase reporter assay kit (Promega, Madison, WI, USA) was used for the determination of relative firefly and renilla luciferase activities in different groups according to the protocol provided by manufacturer. Firefly luciferase activity acted as a control.

Reverse transcription-quantitative PCR (RT-qPCR). Total RNA was extracted from cultured cells using TRIzol (Invitrogen) according to the manufacturer's instructions, miScript SYBR-Green PCR kit (Qiagen, Valencia, CA, USA) was used for the RT-qPCR analysis, which was conducted on an Applied Biosystems 7900HT fast real-time PCR system (Applied Biosystems, Foster City, CA, USA). U6 acted as a control for miR-98 and GAPDH acted as a control for MAPK6. Primers were as: MAPK6 forward, 5'-TAAAGCCAT TGACATGTGGG-3' and reverse, 5'-TCGTGCACAACAGGG ATAGA-3'; GAPDH forward, 5'-ACAAGATGGTGAAGG TCGGTGTGA-3' and reverse, 5'-AGCTTCCCATTCTCA GCCTTGACT-3'; miR-98, 5'-CCGAGGTAGTAAGTTGTA TTGTT-3'; U6, 5'-ACGCAAATTCGTGAAGCGTT-3'. At last, relative level of hsa-miR-98 and MAPK6 in cells was quantified with the method of $2^{-\Delta \Delta C T}$.

Cell apoptosis assay. At $48 \mathrm{~h}$ after cell transfection, HUVECs were harvested and washed with PBS for three times. HUVEC cells were added with $5 \mu \mathrm{l}$ Annexin V-FITC and $5 \mu \mathrm{l}$ PI, stained in the dark for 15 min with Annexin V-FITC apoptosis detection kit (KeyGEN Biotech, Nanjing, China). Relative rate of Annexin V-FITC or PI-positive HUVECs in each group was analyzed by flow cytometry.

Cell proliferation assays. MTT assay (Sigma) was adopted for the analysis of cell proliferation of HUVECs in each group.

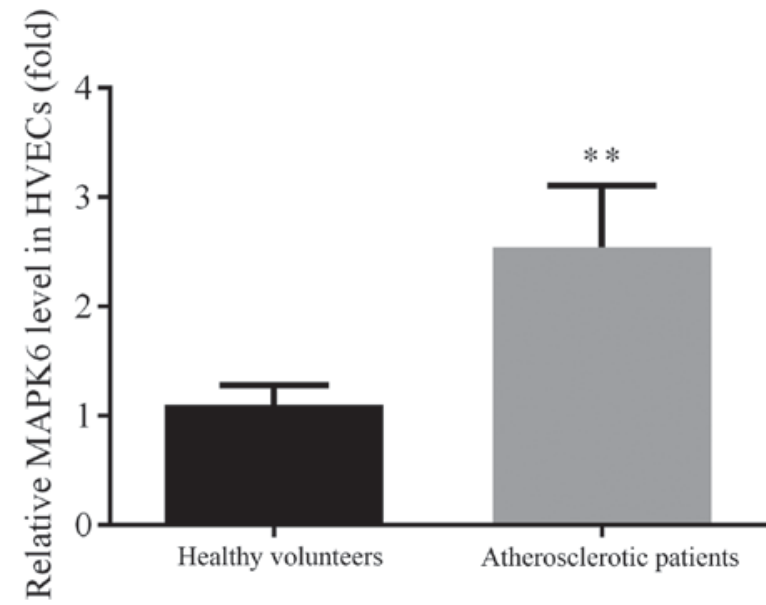

Figure 1. MAPK6 was elevated in the HVECs of atherosclerotic patients compared with healthy volunteers. Expression levels of MAPK6 were detected by RT-qPCR, which indicated a significantly higher MAPK6 mRNA level in HVECs from patients with atherosclerosis compared with healthy volunteers. MAPK6, mitogen activated protein kinase 6; HVECs, human vascular endothelial cells. ${ }^{* *} \mathrm{P}<0.01$

HUVECs were first seeded in 96-well plates and transfected with different plasmids, at $24 \mathrm{~h}$ post-transfection, $25 \mu \mathrm{l}$ MTT $(5 \mathrm{mg} / \mathrm{ml})$ was added into each well, plates were incubated at $37^{\circ} \mathrm{C}$ in an incubator for $3 \mathrm{~h}$. Precipitates in each well were solubilized by $150 \mu 1$ dimethyl sulfoxide (DMSO), assessed at $480 \mathrm{~nm}$ with versaMax ELISA microplate reader (Molecular Devices, Sunnyvale, CA, USA).

Western blotting. Proteins were extracted from HUVECs by RIPA lysis buffer (Invitrogen) and separated by $12 \%$ sodium dodecyl sulfate poly-acrylamide gel electrophoresis before transferring to polyvinylidene fluoride membranes (Millipore, Danvers, MA, USA). After blocking with 5\% nonfat milk at $37^{\circ} \mathrm{C}$ for $2 \mathrm{~h}$, membranes were incubated with the primary antibodies [Bax (dilution, 1:1,000), Bcl-2 (dilution, 1:1,000), caspase-3 (dilution, 1:1,000), (all from Cell Signaling Technology), MAPK6 (dilution, 1:500) and GAPDH (dilution, 1:1,000) (both from Proteintech, Rosemont, IL, USA)] overnight at $4^{\circ} \mathrm{C}$, then HRP-linked secondary antibodies for $2 \mathrm{~h}$ at room temperature. At last, protein bands were visualized by enhanced chemiluminescence (ECL) (General Electric Healthcare, Aurora, OH, USA). GAPDH was used as a control.

Statistical analysis. Data were analyzed by SPSS 17.0 (SPSS, Chicago, IL, USA) with Student's t-test and ANOVA analyses. Results were expressed as mean \pm standard deviation. $\mathrm{P}<0.05$ was considered as statistically significant.

\section{Results}

Different expression levels of MAPK6 in the HVECs of healthy volunteers and atherosclerotic patients. MAPK6 modulates the migration, proliferation and angiogenesis of primary HUVECs (15). Therefore, the expression levels of MAPK6 in HVECs from healthy individuals and subjects with atherosclerosis were detected by RT-qPCR. The expression level of MAPK6 was found to be significantly upregulated in 
C hsa-miR-98/MAPK6 Alignment

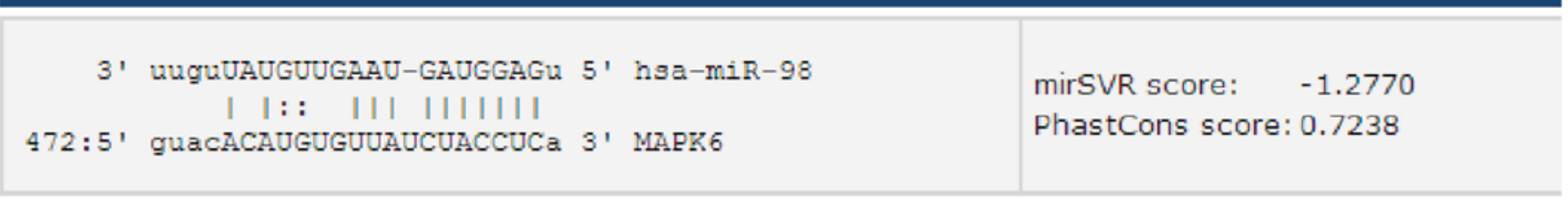

Figure 2. hsa-miR-98 was predicted to target MAPK6. A potential seed sequence of hsa-miR-98 was exhibited in MAPK6-3'-UTR which was identified by miRanda. miR, microRNA; MAPK6, mitogen activated protein kinase.

the HVECs of patients with atherosclerosis when compared with HVECs from healthy volunteers $(\mathrm{P}<0.01$; Fig. 1).

Prediction of the targets of hsa-miR-98. Mounting evidence suggests that, in cardiovascular biology, miRNAs have the ability to regulate gene transcriptional decay and repression by binding target miRNAs (17). A bioinformatic analysis of miRanda was carried out to identify potential miRNAs that target MAPK6, which exhibited a potential seed sequence of hsa-miR-98 in MAPK6-3'-UTR (Fig. 2).

Different expression levels of miR-98 in the HVECs of healthy volunteers and atherosclerotic patients. As acknowledged, miRNAs serve pivotal roles in atherosclerosis, and hsa-miR-98 which was reported to be aberrantly expressed in the serum of atherosclerotic patients was selected for the current research according to the results in a microarray analysis (18). The expression levels of hsa-miR-98 in the HVECs from healthy and atherosclerotic subjects were investigated by analysis using RT-qPCR. The expression of hsa-miR-98 was found to be significantly downregulated in the HVECs from atherosclerotic patients compared with the healthy volunteers $(\mathrm{P}<0.01$; Fig. 3).

Effects of overexpression of miR-98 on cell proliferation. To investigate the potential influences of hsa-miR-98 on cell proliferation, HUVECs were treated with hsa-miR-98 mimics or miR-NC mimic at the concentration of $50 \mathrm{nmol} / 1$ for $48 \mathrm{~h}$. Thereafter, MTT assay was used to assess the influences of hsa-miR-98 on HUVEC proliferation. The results showed that cells transfected with hsa-miR-98 mimics grew much more slowly than cells in the control group and miR-NC mimic group $(\mathrm{P}<0.01$; Fig. 4$)$.

Effects of overexpression of miR-98 on cell apoptosis. To explore the functions of hsa-miR-98 in HUVECs, the cells were transfected with $100 \mathrm{nmol} / \mathrm{l}$ hsa-miR-98 mimic or miR-NC mimic and cultured for $48 \mathrm{~h}$. Afterwards, flow cytometry assay of cell apoptosis was carried out which demonstrated that overexpression of hsa-miR-98 in HUVECs induced an apoptosis rate higher than in those in the control group and miR-NC mimic group $(\mathrm{P}<0.01$; Fig. 5).

Effects of overexpression of miR-98 on Bax, Bcl-2, caspase-3 and MAPK6 protein levels. Western blotting was performed to explore the protein levels of Bax, Bcl-2, caspase-3 and MAPK6 following the administration of hsa-miR-98 mimic or miR-NC mimic. We found that the levels of MAPK6 and Bcl-2

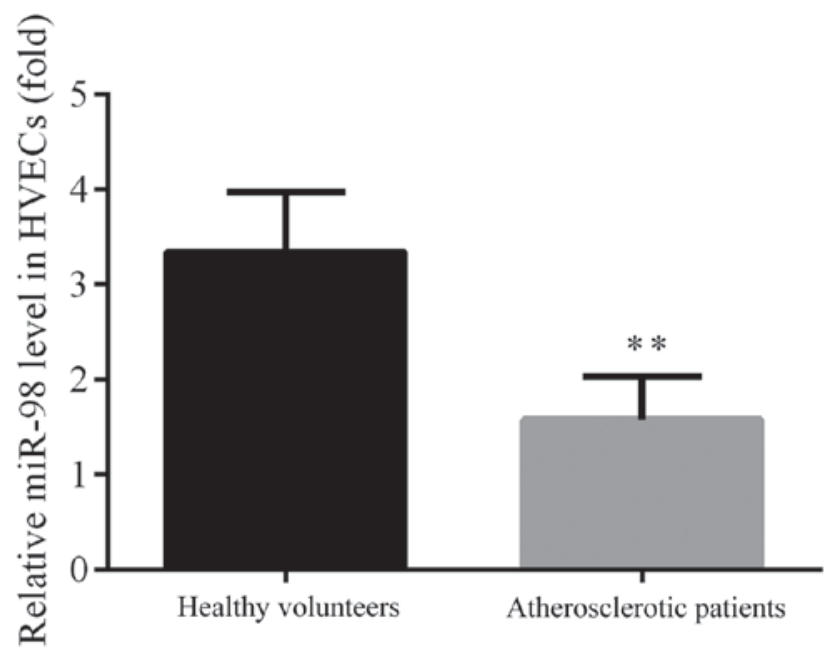

Figure 3. miR-98 was decreased in the HVECs of atherosclerotic patients compared with healthy volunteers. Expression levels of hsa-miR-98 were investigated by RT-qPCR, which demonstrated lower expression of hsa-miR-98 in the HVECs of patients with atherosclerosis compared with healthy individuals. miR, microRNA; HVECs, human vascular endothelial cells. ${ }^{* *} \mathrm{P}<0.01$

were lower, while those of Bax and caspase- 3 were higher in the hsa-miR-98 mimic group compared with the control group and miR-NC mimic group (Fig. 6).

Verification of an interaction between miR-98 and MAPK6. Luciferase assays showed that miR-98 repressed wild-type (WT) MAPK6-3'-UTR-luciferase activity but not mutant MAPK6-3'-UTR-luciferease reporter. This result verified that hsa-miR-98 targeted MAPK6 in HUVECs (Fig. 7).

\section{Discussion}

Atherosclerosis presents as arterial wall thickening $(1,2)$. Endothelial dysfunction occurs initially during the progression of atherosclerosis (19), and is followed by the proliferation and migration of smooth muscle cells (20).

MAPK6 modulates the cell proliferation and angiogenesis of primary HUVECs, and maintains the physiological condition of VSMC (15), whereas its knockdown damages the tube formation of VSMC (16). We first examined MAPK6 mRNA levels in HVECs from healthy and atherosclerotic subjects, and found that MAPK6 was significantly higher in the HVECs of patients with atherosclerosis compared with those of healthy volunteers (Fig. 1). 


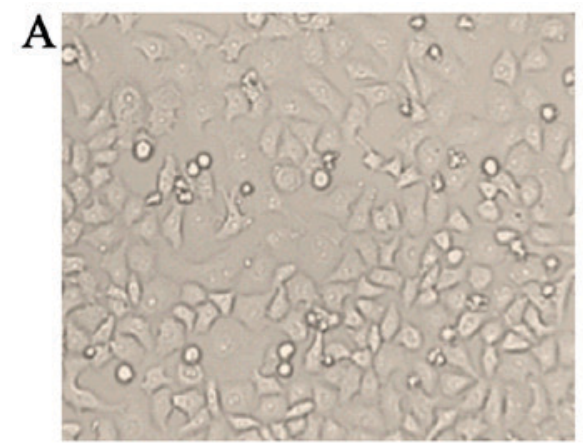

Control

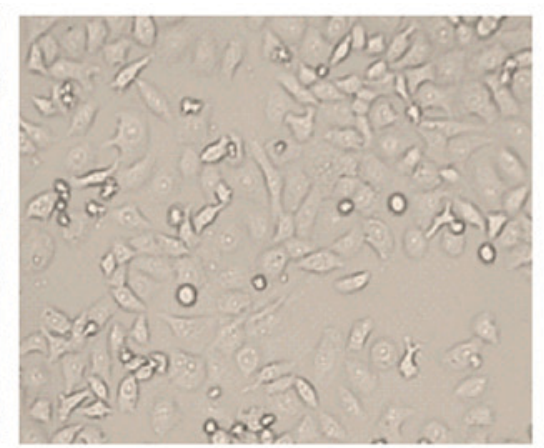

miR-NC mimic

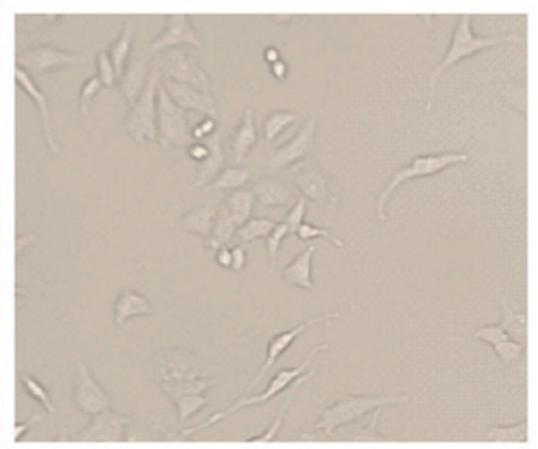

miR-98 mimic

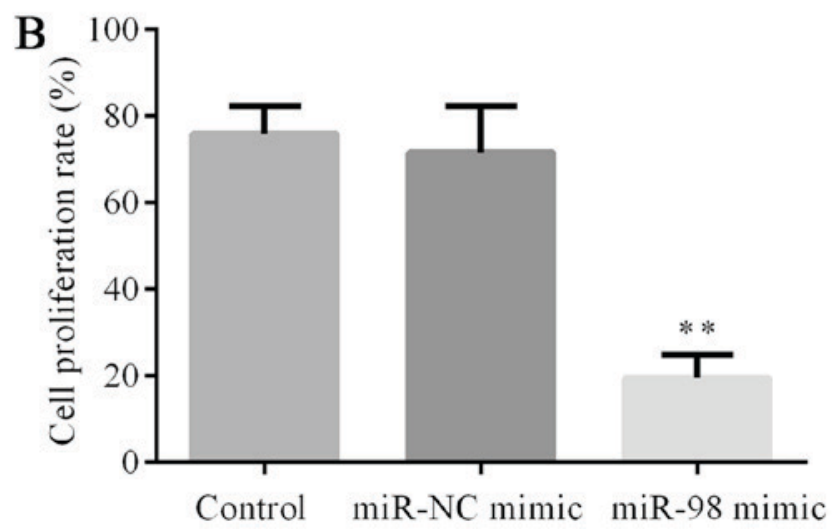

Figure 4. miR-98 mimic repressed the cell proliferation of HUVECs. MTT assay showed slower growth of HUVECs transfected with hsa-miR-98 mimic than cells in the control group and miR-NC mimic group (A and B). "* $\mathrm{P}<0.01$ miR-98 mimic vs. control group and miR-NC mimic. miR, microRNA; HUVECs, human umbilical vein endothelial cells; $\mathrm{NC}$, negative control.

A

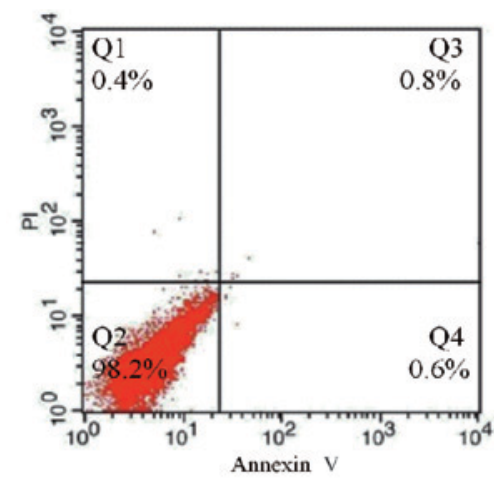

Control

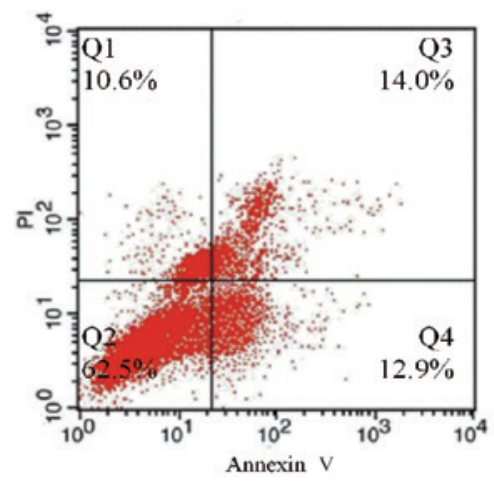

miR-98 mimic

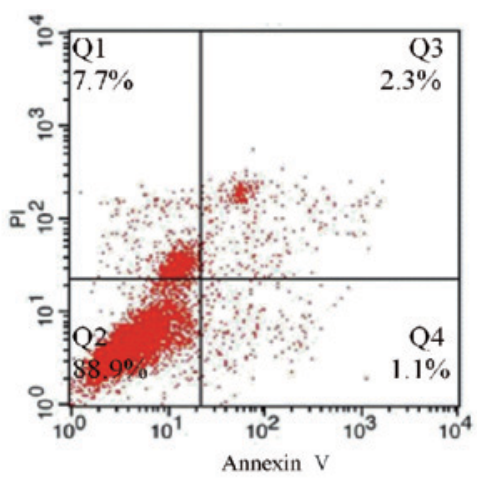

miR-NC mimic

\section{B}

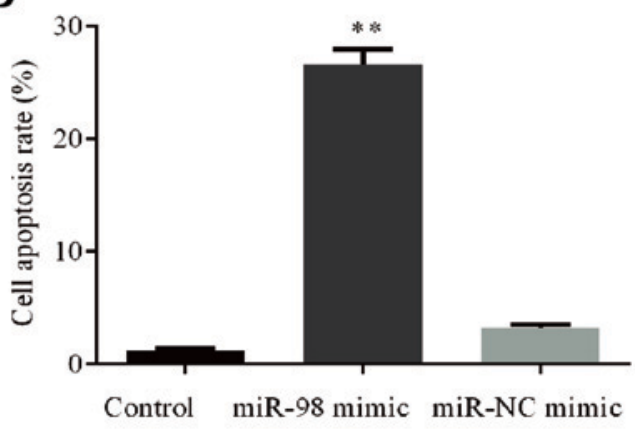

Figure 5. miR-98 mimic induced the cell apoptosis of HUVECs. Flow cytometry assay demonstrated higher apoptosis of HUVECs transfected with hsa-miR-98 than cells in control group and miR-NC mimic group (A and B). ${ }^{* *} \mathrm{P}<0.01$ miR-98 mimic vs. control group and miR-NC mimic. miR, microRNA; HUVECs, human umbilical vein endothelial cells; NC, negative control. 


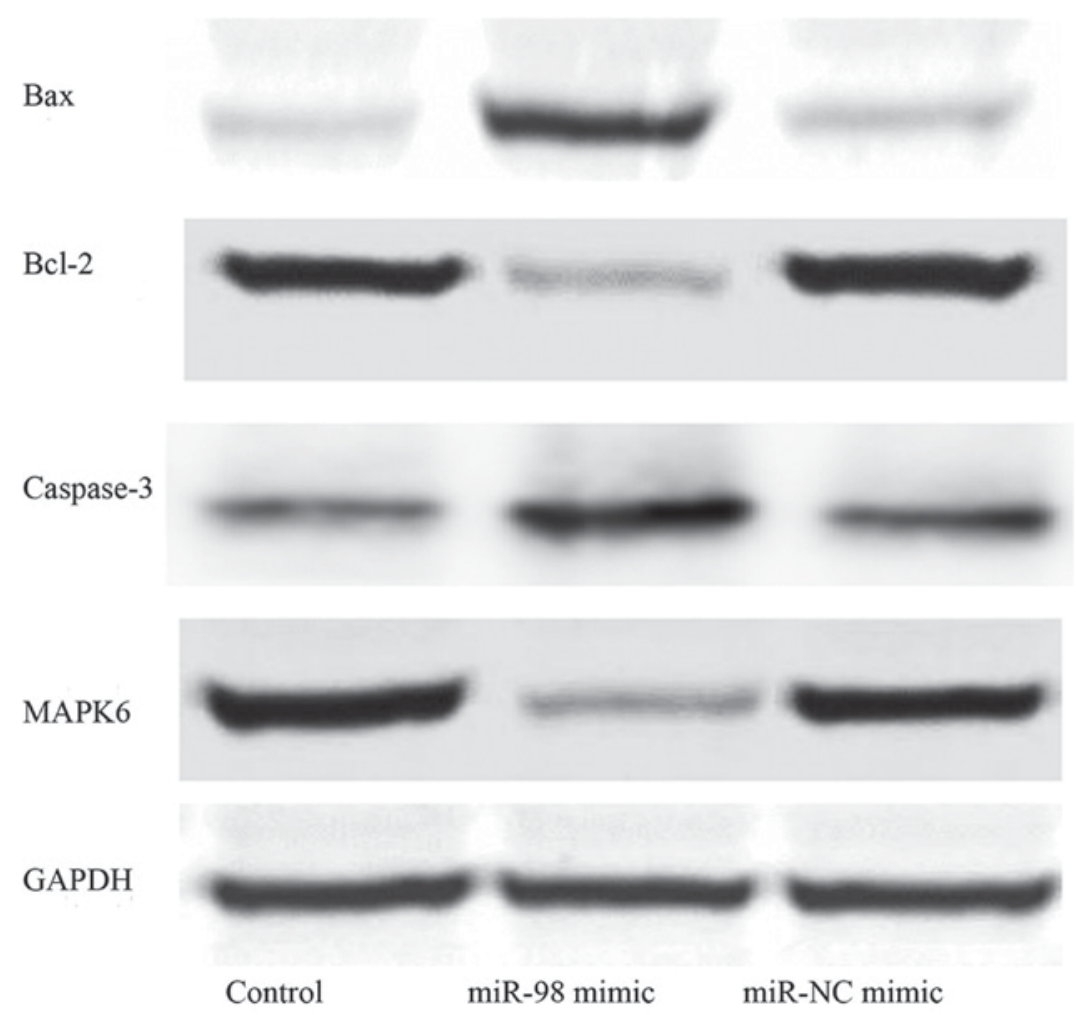

Figure 6. Effects of miR-98 mimic on Bax, Bcl-2, caspase-3 and MAPK6 protein levels. Western blotting exhibited lower protein levels of MAPK6 and Bcl-2 and higher protein levels of Bax and caspase-3 in the hsa-miR-98 mimic group than in the control group and the miR-NC mimic group. miR, microRNA; MAPK, mitogen activated protein kinase; $\mathrm{NC}$, negative control.

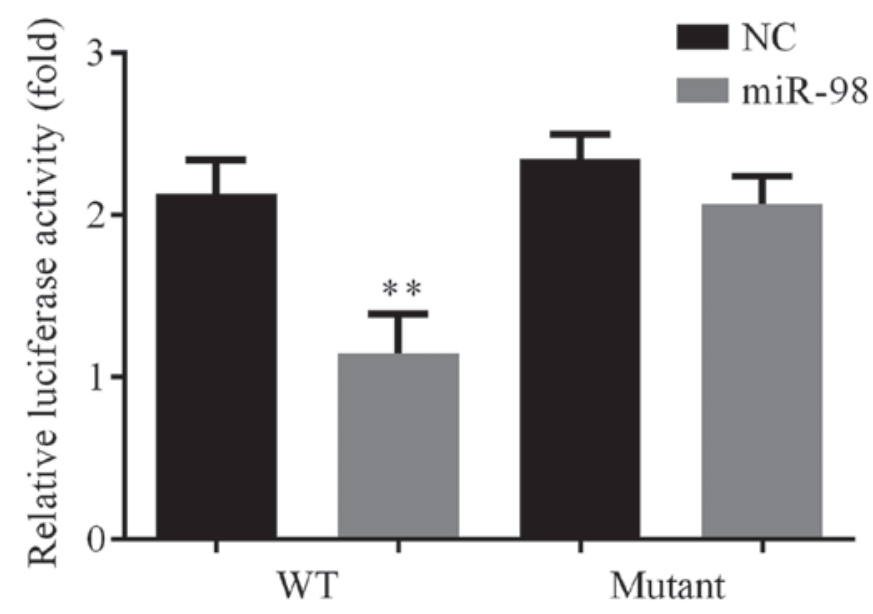

Figure 7. miR-98 was verified to target MAPK6. Lower luciferase activity was found in the WT MAPK6-3'-UTR group but not mutant MAPK6-3'-UTR group, which suggested that hsa-miR-98 targeted MAPK6 in HUVECs. miR, microRNA; MAPK, mitogen activated protein kinase; WT, wild-type; NC, negative control; HUVECs, human vascular endothelial cells. ${ }^{* *} \mathrm{P}<0.01$.

mRNAs have the ability to bind to the 5'UTR or 3'UTR of target genes to inhibit their expression (5-7). Circulating miRNAs are potentially functional in the atherosclerosis associated dysfunction of gene regulatory networks (21) as well as biological processes, for instance, cell proliferation (10). However, it remains unclear whether MAPK6 could be regulated by miRNA in the functioning of the vascular endothelium.
miRanda was adopted to identify miRNAs that could target MAPK6, and a potential seed sequence of hsa-miR-98 was exhibited in the MAPK6-3'-UTR (Fig. 2). Thereafter, we identified the expression levels of hsa-miR-98 in HVECs from healthy and atherosclerotic subjects, and found that hsa-miR-98 was significantly lower in the HVECs of patients with atherosclerosis compared with healthy individuals (Fig. 3), which was consistent with a previous study (18).

The potential influences of hsa-miR-98 on HUVEC cell proliferation and apoptosis were tested by MTT assay and flow cytometry assay, respectively. Results showed slower growth (Fig. 4) and a higher apoptotic rate (Fig. 5) in HUVECs transfected with hsa-miR-98 mimic than in control group and miR-NC mimic group.

Bcl-2 and caspase families participate in the apoptotic process. Bcl-2 protein families include Bax (pro-apoptotic) and Bcl-2 (antiapoptotic) (22). Moreover, since caspase-3 is the most reliable determining factor during apoptosis (23), Bcl-2 regulates apoptosis via a caspase-3 dependent pathway (24). Western blotting results showed that MAPK6 and Bcl-2 levels were lower, while Bax and caspase- 3 levels were higher in the hsa-miR-98 mimic group than in control group and miR-NC mimic group (Fig. 6).

Finally, the interaction between miR-98 and MAPK6 was tested by luciferase assay. The results verified that hsa-miR-98 targeted MAPK6 in HUVECs (Fig. 7).

In conclusion, miR-98 inhibited HUVEC proliferation, promoted HUVEC apoptosis, lowered MAPK6/Bcl-2 and upregulated Bax/caspase-3 via targeting MAPK6. Thus miR-98 might be a therapeutic target for atherosclerosis. 


\section{References}

1. Ross R: The pathogenesis of atherosclerosis: A perspective for the 1990s. Nature 362: 801-809, 1993.

2. Hansson GK and Hermansson A: The immune system in atherosclerosis. Nat Immunol 12: 204-212, 2011.

3. Lüscher TF and Barton M: Biology of the endothelium. Clin Cardiol 20 (11 Suppl 2): II-3-10, 1997.

4. Olejarz W, Bryk D, Zapolska-Downar D, Małecki M, Stachurska A and Sitkiewicz D: Mycophenolic acid attenuates the tumour necrosis factor $\alpha$-mediated proinflammatory response in endothelial cells by blocking the MAPK/NF- $\mathrm{KB}$ and ROS pathways. Eur J Clin Invest 44: 54-64, 2014.

5. Pillai RS, Bhattacharyya SN and Filipowicz W: Repression of protein synthesis by miRNAs: How many mechanisms? Trends Cell Biol 17: 118-126, 2007.

6. Forman JJ and Coller HA: The code within the code: microRNAs target coding regions. Cell Cycle 9: 1533-1541, 2010.

7. Lytle JR, Yario TA and Steitz JA: Target mRNAs are repressed as efficiently by microRNA-binding sites in the 5' UTR as in the 3'UTR. Proc Natl Acad Sci USA 104: 9667-9672, 2007.

8. McManus DD and Ambros V: Circulating MicroRNAs in cardiovascular disease. Circulation 124: 1908-1910, 2011.

9. Kosaka N, Iguchi H and Ochiya T: Circulating microRNA in body fluid: A new potential biomarker for cancer diagnosis and prognosis. Cancer Sci 101: 2087-2092, 2010.

10. Cirera-Salinas D, Pauta M, Allen RM, Salerno AG, Ramírez CM, Chamorro-Jorganes A, Wanschel AC, Lasuncion MA, Morales-Ruiz M, Suarez Y, et al: mir-33 regulates cell proliferation and cell cycle progression. Cell Cycle 11: 922-933, 2012.

11. Hwang HW and Mendell JT: MicroRNAs in cell proliferation, cell death, and tumorigenesis. Br J Cancer 94: 776-780, 2006.

12. Chen WJ, Yin K, Zhao GJ, Fu YC and Tang CK: The magic and mystery of microRNA-27 in atherosclerosis. Atherosclerosis 222: 314-323, 2012.

13. Torella D, Iaconetti C, Catalucci D, Ellison GM, Leone A, Waring CD, Bochicchio A, Vicinanza C, Aquila I, Curcio A, et al: MicroRNA-133 controls vascular smooth muscle cell phenotypic switch in vitro and vascular remodeling in vivo. Circ Res 109: 880-893, 2011.
14. Meloche S, Beatty BG and Pellerin J: Primary structure, expression and chromosomal locus of a human homolog of rat ERK3. Oncogene 13: 1575-1579, 1996.

15. Tan J, Yang L, Liu C and Yan Z: MicroRNA-26a targets MAPK6 to inhibit smooth muscle cell proliferation and vein graft neointimal hyperplasia. Sci Rep 7: 46602, 2017.

16. Tak H, Jang E, Kim SB, Park J, Suk J, Yoon YS, Ahn JK, Lee JH and Joe CO: 14-3-3epsilon inhibits MK5-mediated cell migration by disrupting F-actin polymerization. Cell Signal 19: 2379-2387, 2007.

17. Small EM and Olson EN: Pervasive roles of microRNAs in cardiovascular biology. Nature 469: 336-342, 2011.

18. Song Z and Li G: Role of specific microRNAs in regulation of vascular smooth muscle cell differentiation and the response to injury. J Cardiovasc Transl Res 3: 246-250, 2010.

19. Mudau M, Genis A, Lochner A and Strijdom H: Endothelial dysfunction: The early predictor of atherosclerosis. Cardiovasc J Afr 23: 222-231, 2012

20. Libby P: Inflammation in atherosclerosis. Nature 420: 868-874, 2002.

21. Fish JE, Santoro MM, Morton SU, Yu S, Yeh RF, Wythe JD, Ivey KN, Bruneau BG, Stainier DY and Srivastava D: MiR-126 regulates angiogenic signaling and vascular integrity. Dev Cell 15: 272-284, 2008.

22. Danial NN and Korsmeyer SJ: Cell death: Critical control points. Cell 116: 205-219, 2004

23. Kaufmann SH, Lee SH, Meng XW, Loegering DA, Kottke TJ, Henzing AJ, Ruchaud S, Samejima K and Earnshaw WC: Apoptosis-associated caspase activation assays. Methods 44: 262-272, 2008

24. Marsden VS, O'Connor L, O'Reilly LA, Silke J, Metcalf D, Ekert PG, Huang DC, Cecconi F, Kuida K, Tomaselli KJ, et al: Apoptosis initiated by $\mathrm{Bcl}-2$-regulated caspase activation independently of the cytochrome c/Apaf-1/caspase-9 apoptosome. Nature 419: 634-637, 2002.

(c) (i) (5) This work is licensed under a Creative Commons Attribution-NonCommercial-NoDerivatives 4.0 International (CC BY-NC-ND 4.0) License. 